\title{
KRDD: Korean Rice Ds-tagging Lines Database for Rice (Oryza sativa L. Dongjin)
}

\author{
Chang Kug Kim ${ }^{1}$, Myung Chul Lee ${ }^{2}$, Byung \\ Ohg Ahn', Doh Won Yun', Ung Han Yoon', \\ Seok Cheol Suh', Moo Young Eun ${ }^{2}$ and Jang \\ Ho Hahn ${ }^{1 *}$
}

${ }^{1}$ Bioinformatics Division, National Institute of Agricultural, Biotechnology (NIAB), Suwon 441-707, Korea, ${ }^{2}$ Cell and Genetics Division, NIAB, Suwon 441-707, Korea

\begin{abstract}
The Korean Rice Ds-tagging lines Database (KRDD) is designed to provide information about Ac/Ds insertion lines and activation tagging lines using japonica rice. This database has provided information on 18,158 Ds lines, which includes the ID, description, photo image, sequence information, and gene characteristics. The KRDD is visualized using a web-based graphical view, and anonymous users can query and browse the data using the search function. It has four major menus of web pages: (i) a Blast Search menu of a mutant line; Blast from rice Ds-tagging mutant lines; (ii) a primer design tool to identify genotypes of Ds insertion lines; (iii) a Phenotype menu for Ds lines, searching by identification name and phenotype characteristics; and (iv) a Management menu for Ds lines.
\end{abstract}

Availability: The KRDD can be accessed at the web site of the National Institute of Agricultural Biotechnology (http://www. niab.go.kr/RDS/)

Keywords: Ac/Ds database, Ds phenotype, Ds-tagging line, insertion line, KRDD

\section{Introduction}

Rice is one of the most important cereal crops in Korea. It has become a model for monocot plants because of its small genome size (around $430 \mathrm{Mbp}$ ), ease of transformation, availability of genetic information, and economic importance (Goff et al., 2002; Keller et al., 2000, Sasaki et al., 2005). In rice, insertion of mutant genes has been a powerful strategy for assessing gene func-

*Corresponding author: E-mail jhhahn@rda.go.kr

Tel +82-31-299-1651, Fax +82-31-299-1642

Accepted 21 March 2008 tion. The large-scale application of databases in T-DNA insertion sites requires considerable effort (Parinov et al., 2000). But this database can easily be shared with the distribution of mutant materials and analysis of end sequence databases for T-DNA tagging lines in rice (An et al., 2003).

The Activator/Dissociation (Ac/Ds) line has a variation in the transposition frequency and distribution pattern. The Ac element was shown to transpose at high somatic and transposition behaviors of an Ac/Ds system in rice (Enoki et al., 1999; Greco et al., 2003). The flanking sequence-tag database showed generation of an activation-tagging line in japonica rice (Jeong et al., 2006). In this study, we obtained data on the characteristic information of the Ac/Ds family by analyzing insertion and activation tagging lines using japonica rice (Oryza sativa L. Dongjin). (Kim et al., 2004; Park et al., 2007).

\section{Results and Discussion}

The KRDD is designed to provide information about the Ac/Ds insertion line and activation tagging lines using japonica rice. This database has provided information on 18,158 Ds lines, including the ID, description, photo image, sequence information, and gene characteristics. Cross-links to other databases are also introduced to facilitate the access to individual clone information. The use of this database is free for non-commercial purposes. The KRDD is visualized using a web-based graphical view, and anonymous users can query and browse the data using the search function.

The KRDD homepage is shown in Fig. 1, and the stored data are visualized using a web-based graphical view. It has four major menus of web pages: (i) a Blast Search of a mutant line; Blast from rice Ds-tagging mutant lines; (ii) a primer design tool to identify genotypes of Ds insertion lines; (iii) a Phenotype menu for Ds lines, searching by gene name and phenotype characteristics among specific Ds lines; and (iv) a Management menu for Ds lines.

The Blast Search is searchable by selecting specific databases, consisting of DS Sequence, Indica Core, Japonica Core, Indica EST, Japonica EST, Indica Genome, Japonica Genome, Indica GSS, and Japonica GSS in Oryza sativa. The KRDD uses several reference databases to facilitate a comprehensive analysis of the genome sequence. These include the Entrez nucleotide database of the National Center for Biotechnology 


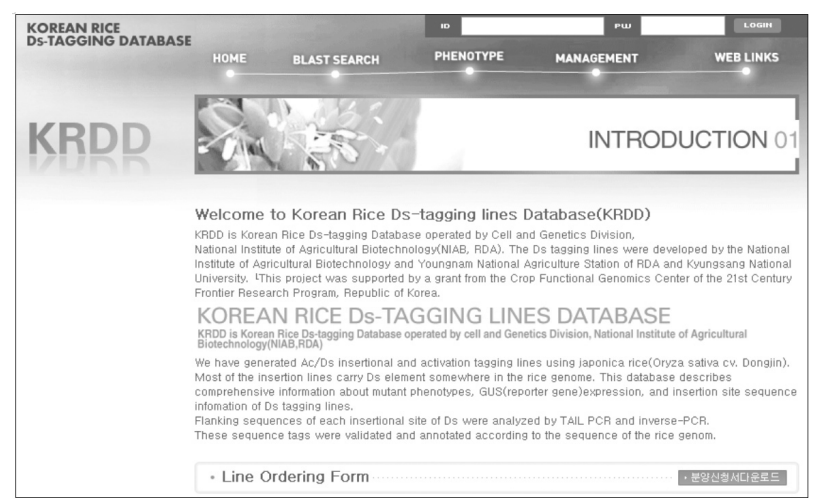

Fig. 1. Homepage of the KRDD. This database shows comprehensive information about mutant phenotypes and sequence information of Ds tagging lines.

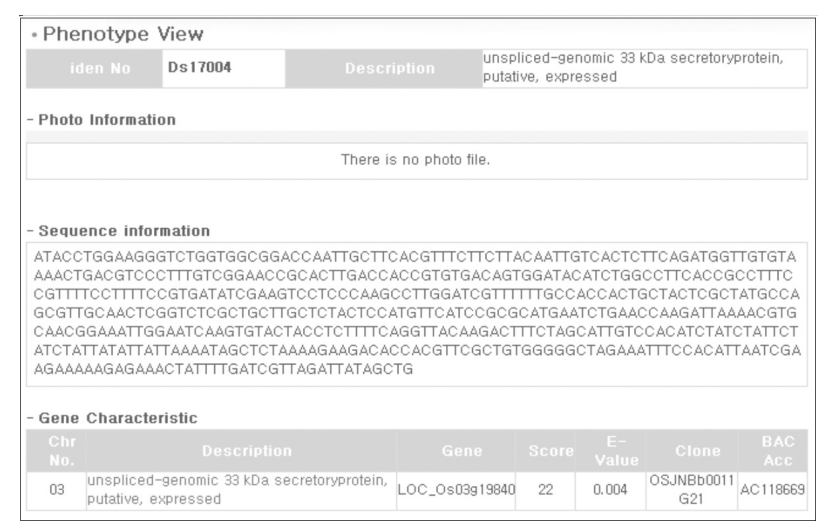

Fig. 2. Typical result page of search for a DS line. The phenotype view page shows Ds line information, including the ID, description, photo information, sequence information, and gene characteristics.

Information (NCBI) for homology searches and Rice Genome Annotation version 5 of the Institute for Genomic Research (TIGR).

The Phenotype View can be searched using Ds sequence information that includes chromosome location (cM) in relation to anchored genetic markers. To integrate genetic maps with Ds sequences, we searched bacterial artificial chromosome (BAC) and P1-derived artificial chromosome (PAC) clones for each chromosome that contained Ds-flanking DNA. As shown in Fig. 2, a phenotype view window shows that all analysis results for a sequence are integrated in the Ds-tagging mutant line. The gene characteristic was designed based on the concept of the Primer design tool throughout the TIGR annotation database. The DS position of chromosome was calculated by the Primer design tool program.

A simplified view of the KRDD data model integrates

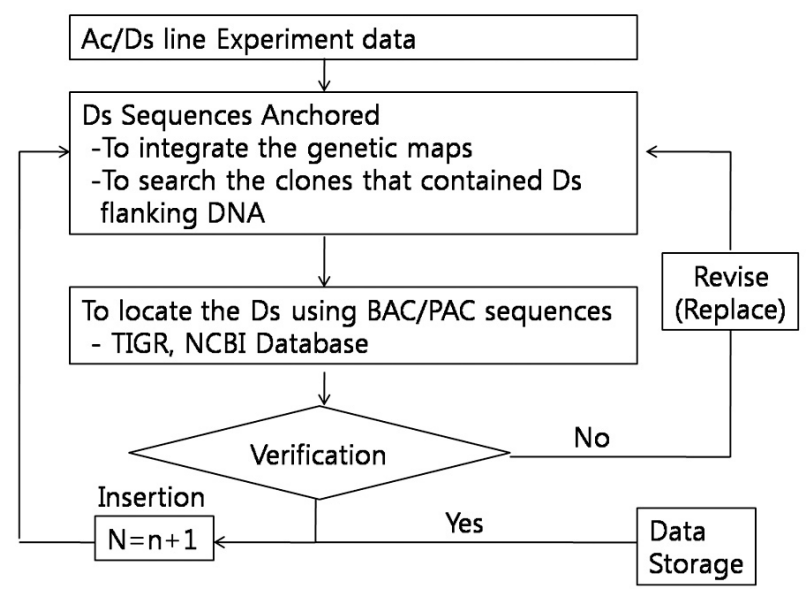

Fig. 3. System flowchart of KRDD. Data collected from users are stored in the KRDD. All registration results are visualized using a web-based graphical view and are made accessible via the Internet.

several programs for search and analysis of Ds line structures (Fig. 3). The physical schema of the database is shown schematically in Fig. 4. It is designed specifically to provide an efficient system and a comprehensive database for Ds lines. It is a very simple model, based on only two core types of objects.

The interface is prepared to submit and analyze each sequence. To prevent disclosure, a user needs to register and enter a password, which serves as security control. The improvement of data quality can be made in the 'Verification' section of the database manager, which allows the user to modify, delete, and add information. The total information on 18,158 Ds lines is collected by the system and then is made available to the public via the Internet. The KRDD will collect additional information and develop a useful tool for Ac/Ds line information.

\section{Acknowledgements}

The Ds tagging lines were supported by the NIAB, Yeongnam Agricultural Research Institute (YARI), and Kyungsang National University. This work was supported by the Rural Development Administration (NIAB 06-2-12-7) and a grant (code CG1510, CG2141) from the Crop Functional Genomics Center of the 21st Century Frontier Research Program of Republic of Korea. This database was developed by an NIAB research project (the construction of an agricultural biotechnology information management system). 


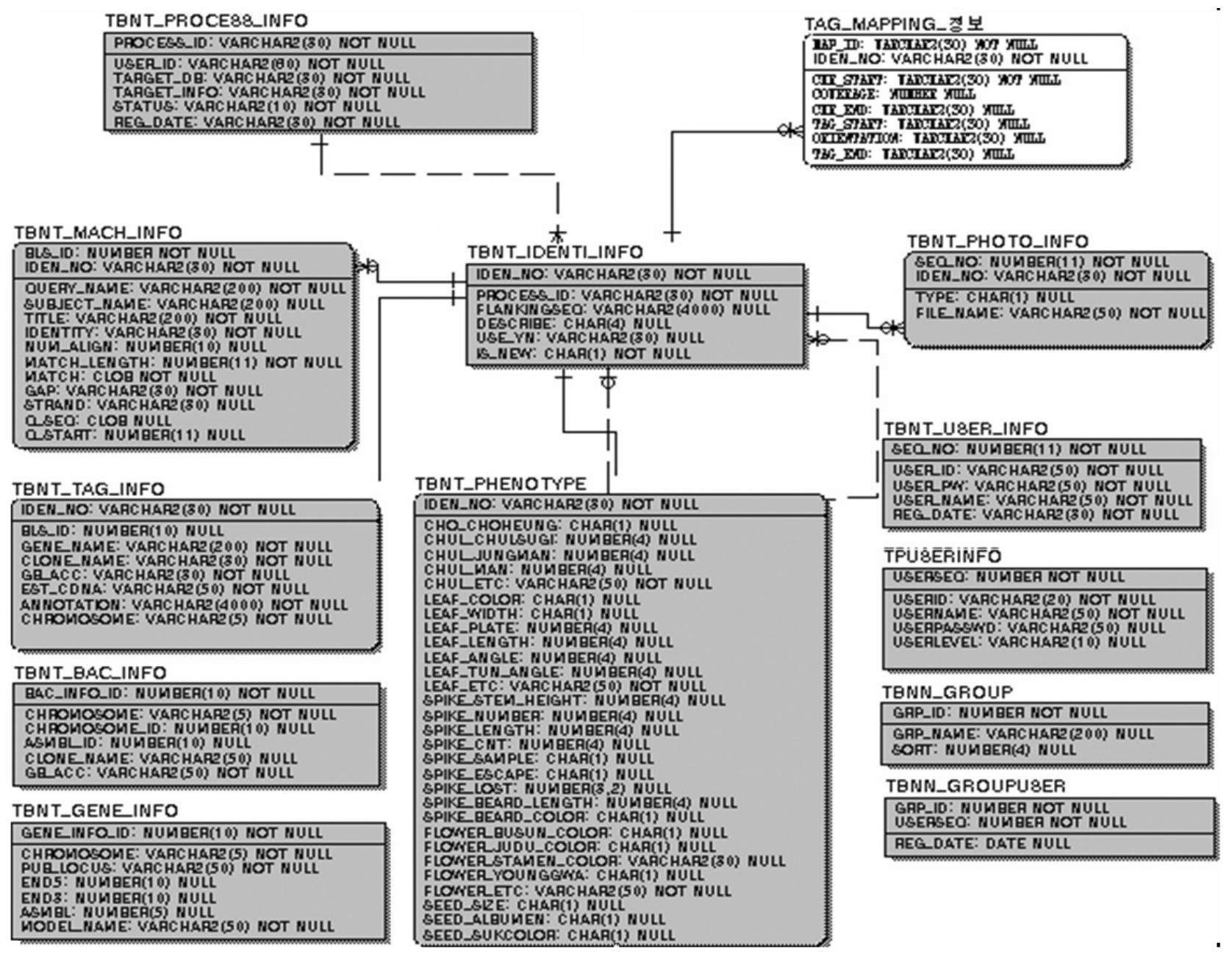

Fig. 4. The physical schema of the database. Each box represents a separate entity, implemented as a database table. Lines between entities indicate a relationship exists. Some tables and columns have been omitted to make the diagram more concise.

\section{References}

An, S., Park, S., Jeong, D.H., et al. (2003). Generation and analysis of end sequence database for T-DNA tagging lines in rice. Plant. Physiology 133, 2040-2047.

Kim, C.M., Piao, H.L., Park, S.J., Chon, N.S., Je, B.I., Sun, B., Park, S.H., Park, J.Y., Lee, E.J., Kim, M.J., Lee, K.H., Lee, Y.S., Lee, J.J., Won, Y.J., Yi, G.H., Nam, M.H., Cha, Y.S., Yun, D.W., Eun, M.Y., and Han, C.D. (2004). Rapid, large-scale generation of $D s$ transposant lines and analysis of the $D s$ insertion sites in rice. Plant J. 39, 252-263.

Jeong, D.H., An, S.Y., Park, S.H., Kang, H.G., Park, G.G., Kim, S.R., Sim, J.Y., Kim, Y.O., Kim, M.K., Kim, J.W., Shin, M.S., Jung, M.Y., and An, G.H. (2006). Generation of a flanking sequence-tag database for activation-tagging lines in japonica rice. Plant J. 45, 123-132.

Enoki, H., Izawa, T., Kawahara, M., Komatsu, M., Koh, S., Kyozuka, J., and Shimamoto, K. (1999). Ac as a tool for the functional genomics of rice. Plant. J. 19, 605-613.

Goff, S.A., Ricke, D., Lan, T.H., et al. (2002). A draft sequence of the rice genome (Oryza sativa L. ssp. japonica). Science 296, 92-100.

Greco, R., Ouwerkerk, P.B., De Kam, R.J., Sallaud, C., Favalli, C., Colombo, L., Guiderdoni, E., Meijer, A.H., Hoge Dagger, J.H., and Pereira, A. (2003). Transpositional behaviour of an Ac/Ds system for reverse genetics in rice. Theor. Appl. Genet. 108, 10-24.

Keller, B., and Feuillet, C. (2000). Colinearity and gene density in grass genomes. Trends. Plant Sci. 5, 246-251.

Parinov, S., and Sundaresan, V. (2000). Functional genomics in Arabidopsis: large-scale insertional mutagenesis complements the genome sequencing project. Curr. Opin. Biotechnol. 11, 157-161.

Sasaki, T., Matsumoto, T., Antonio, B.A., and Nagamura, Y. (2005). From mapping to sequencing, post-sequencing and beyond. Plant. Cell Physiol. 46, 3-13.

Park, S.H., Jun, N.S., Kim, C.M., Oh, T.Y., Huang, J., Xuan, 
Y.J., Park, S.J., Je, B.I., Piao, H.L., Park, S.H., Cha, Y.S., Ahn, B.O., Ji, H.S., Lee, M.C., Suh, S.C., Nam, M.H., Eun, M.Y., Yi, G.H., Yun, D.W., and Han. C.D.
(2007). Analysis of gene-trap Ds rice populations in Korea. Plant Molecular Biology 65, 373-384 